\title{
ANÁLISE DO PERFIL SOCIODEMOGRÁFICO DE PACIENTES SUBMETIDOS AO TRATAMENTO DE TUBERCULOSE NO MUNICÍPIO DO RECIFE
}

\author{
L. D. SANTANA ${ }^{1}$, F. L. A. SANTOS ${ }^{2}$, J. M. ALMEIDA ${ }^{2}$, J. L. N. NASCIMENTO ${ }^{1}$, P. J. \\ ROLIM NETO ${ }^{1}$ e R. M. F. SILVA ${ }^{1}$
}

${ }^{1}$ Universidade Federal de Pernambuco, Centro de Ciências da Saúde, Departamento de Ciências Farmacêuticas

${ }^{2}$ Prefeitura do Recife, Distrito Sanitário VI.

E-mail para contato: jlnsmto@gmail.com

\begin{abstract}
RESUMO - Dentre as doenças endêmicas que acometem o Brasil, a tuberculose é de notificação compulsória, tendo como agente etiológico Mycobacterium tuberculosis. $O$ delineamento de um perfil sociodemográfico visa identificar as necessidades de uma população por ele estudada e assim avaliar os investimentos na saúde da população. O presente trabalho visou uma análise do perfil sóciodemográfico do Distrito Sanitário VI da Prefeitura do Recife, com intuito de analisar as características sociais e demográficas das populações acolhidas pelo programa de tuberculose. Trata-se de um estudo retrospectivo, em que os dados corresponderam ao periodo de janeiro a dezembro de 2016, e os mesmos foram coletados no acervo da Farmácia do Distrito Sanitário VI, bem como do sistema Hórus ${ }^{\circledR}$. Foi observado um total de 88 fichas de notificação para usuários do programa de tuberculose. Observou-se nesses pacientes uma maior incidência de pacientes do sexo masculino com idade na faixa etária entre seus 20-29 anos, cor parda, residentes em Brasília Teimosa e que não declararam a ocupação. Estudos realizados no Distrito Sanitário VI da Prefeitura do Recife ainda são escassos, podendo este trabalho servir como incentivo a outros para contribuir com a melhoria da assistência farmacêutica no município.
\end{abstract}

Palavras-chave: Doença endêmica. Distrito sanitário. Características demográficas.

ABSTRACT - Among the endemic diseases that affect Brazil, tuberculosis is compulsory, with Mycobacterium tuberculosis as the etiological agent. The outline of a sociodemographic profile aims to identify the needs of a population studied by it and thus evaluate the investments in the health of the population. The present work aimed at analyzing the social and demographic profile of the Sanitary District VI of the City Hall of Recife, in order to analyze the social and demographic characteristics of the populations hosted by the tuberculosis program. This is a retrospective study, in which the data corresponded to the period from January to December 2016, and were collected in the collection of the pharmacy of the Sanitary District VI as well as the Horus ${ }^{\circledR}$ system. A total of 88 notification sheets were observed for users of the tuberculosis program. In these patients, a higher incidence of male patients aged 20-29 years, brown, 
residing in Brasilia Teimosa and who did not declare the occupation was observed. Studies performed in the Health District VI of the City Hall of Recife are still scarce, and this work may serve as an incentive to others to contribute to the improvement of pharmaceutical care in the municipality.

Key words: Endemic disease. Sanitary District. Demographic characteristics.

\section{INTRODUÇÃO}

No Brasil, a tuberculose é uma das principais doenças endêmicas, sendo a tuberculose sujeita à notificação compulsória semanalmente - notificação compulsória realizada em até 7 (sete) dias, a partir do conhecimento da ocorrência de doença ou agravo (BRASIL, 2014). Segundo a Secretaria de Saúde, tuberculose é uma doença infectocontagiosa que tem como agente etiológico a bactéria Mycobacterium tuberculosis ou Bacilo de Koch (BK), que afeta principalmente os pulmões, embora possa ocorrer em outros órgãos do corpo. A tuberculose está intimamente ligada à pobreza e à má distribuição de renda, além do estigma que implica na não adesão dos portadores e/ou familiares/contactantes. Além disso, outros fatores podem estar associados como baixa renda familiar, educação precária, habitação precária/inexistente, famílias numerosas, adensamentos comunitários, má nutrição, alcoolismo e outras doenças infecciosas associadas (COÊLHO et al., 2010). A Prefeitura do Recife é constituída de oito Distritos Sanitários (DS), onde cada Distrito compreende bairros de acordo com a divisão geográfica. O Distrito objeto do presente estudo foi o DS VI que compreende os bairros de Boa Viagem, Brasília Teimosa, Imbiribeira, Ipsep e Pina. Analisar um perfil sóciodemográfico em um Distrito Sanitário objetiva fornecer informações capazes de orientar as políticas públicas, em especial a política de atenção à saúde, e subsidiar o planejamento das ações a serem desenvolvidas.

\section{MATERIAIS E MÉTODOS}

O presente trabalho trata-se de um estudo retrospectivo, de natureza quantitativa, de cunho experimental-social realizado na Farmácia do Distrito Sanitário VI (DS VI), da Prefeitura do Recife. Os dados necessários para o estudo foram obtidos a partir da análise de fichas de notificação que constam no acervo da Farmácia do DS VI, e análise no sistema HÓRUS $^{\circledR}$, desenvolvido pela Prefeitura do Recife. As informações coletadas corresponderam a 88 fichas de notificações para o programa de tuberculose, referentes ao período de janeiro a dezembro de 2016. Foram excluídos aqueles dados cujas informações estavam incompletas ou ilegíveis, bem como casos de dupla notificação. Após o tratamento, as informações foram dispostas em forma de gráficos gerados por Microsoft Excel $^{\circledR}$, para melhor análise dessas informações. O projeto foi submetido e aprovado ao Comité de Ética em Pesquisa (CEP) em humanos, da Universidade Federal de Pernambuco, recebendo o número CAAE: 75671417.0.0000.5208.

\section{RESULTADOS E DISCUSSÃO}

Pôde-se observar que 59\% $(n=52)$ referiram-se a pacientes do sexo masculino e $41 \%$ $(n=36)$ foram do sexo feminino. A idade média foi de 34,6 anos, em que o maior percentual ficou na faixa etária dos 20-29 anos, e o menor percentual na faixa de 70-80 anos. Esses resultados também foram observados em um estudo realizado em um centro de tratamento de tuberculose no estado de Ogun, Nigéria com pacientes com tuberculose resistente, onde 
eles demonstraram uma idade média de 34,5 (SOGEBI et al, 2017). Também foi verificado que cerca de $47 \%(n=41)$ dos pacientes se declararam de raça parda (Figura 1) e residiam, em sua maioria, no bairro de Brasília Teimosa e a minoria no bairro do Pina. Na (Figura 1) podemos verificar que a maioria dos pacientes $(26 \%)$ possuía o ensino médio, sendo este completo ou cursando. Uma possível explicação seria a diminuição que o país sofre na taxa de analfabetismo em pessoas maiores de 15 anos, com índice estimado em 8\% da população, segundo dados do IBGE, 2016.

Figura 1 - Caracterização da variável sociodemográfica "Raça e Nível de Escolaridade".

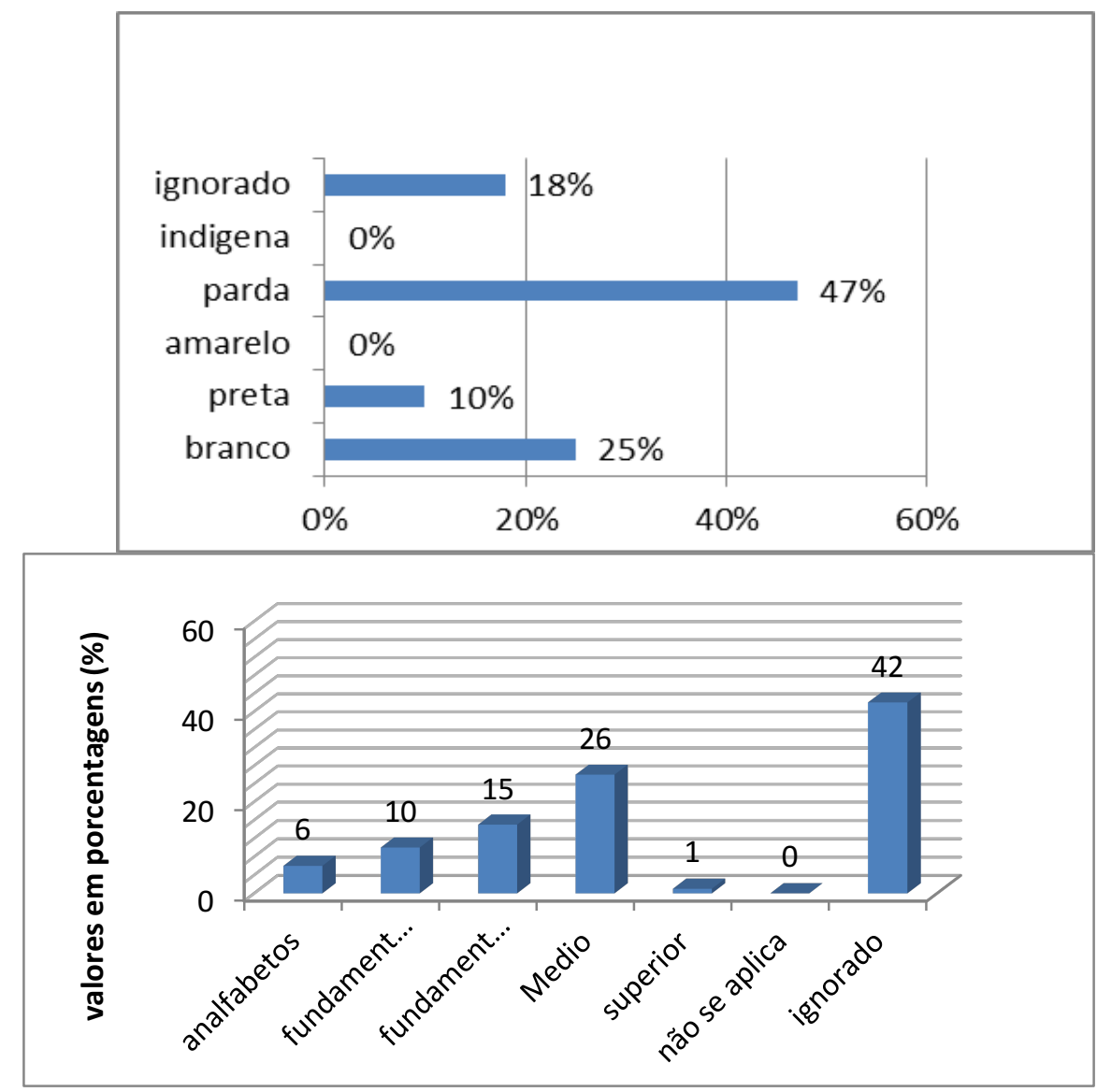

Entre as fichas de notificação analisadas, verificou-se que a maioria destas não foi devidamente preenchida, sendo apresentadas no presente trabalho como variável ignorada. $\mathrm{Na}$ (tabela 1) podemos observar também que, nestas fichas, o campo de ocupação só foi preenchido para 19 pacientes, sendo a maioria declarada como pedreiro, estudante e donas de casa $(n=3)$. 
Tabela 1- Caracterização da variável sociodemográfica "Ocupação"

\begin{tabular}{lcc}
\hline VARIÁVEIS & N & \% \\
\hline Ocupação & & \\
Militar & 1 & 1 \\
Tatuador & 1 & 1 \\
Eletricista & 2 & 2 \\
Dona de casa & 3 & 3 \\
Estudante & 3 & 3 \\
Pedreiro/Ajudante de pedreiro & 3 & 3 \\
Pensionista & 1 & 1 \\
Catador & 1 & 1 \\
Universitário & 1 & 1 \\
Caixa de supermercado & 1 & 1 \\
Prof. De saúde & 1 & 1 \\
Desempregado & 1 & 1 \\
lgnorado & 69 & 78 \\
\hline TOTAL & $\mathbf{8 8}$ & $\mathbf{1 0 0}$ \\
\hline
\end{tabular}

\section{CONCLUSÃO}

O presente trabalho analisou o perfil social e demográfico, a partir das fichas de notificação do programa governamental de tuberculose. Foi observado que as fichas para esta doença tendem a estar incompletas quanto ao seu preenchimento. As variáveis: agravos associados e relação da doença com trabalho não foram devidamente preenchidas nas fichas, embora pudessem ser uma nova vertente de futuros estudos. Devem ser feitas ações nos bairros de Brasília Teimosa e Imbiribeira para promover a prevenção e evitar o contagio dessa doença, e se ter uma maior atenção em relação ao preenchimento dos formulários. Além de acompanhar constantemente o paciente com tuberculose, avaliar a utilização de medicamentos para evitar usos incorretos é, também, dever do farmacêutico informar à população sobre a transmissão, orientar sobre as formas de prevenção da doença e, ainda, educar a população e informar aos demais profissionais que compõe as equipes de saúde sobre o uso racional de medicamentos. Com isso pode-se diminuir o abandono do tratamento da tuberculose e ainda evitar o contágio. Estudos realizados no Distrito Sanitário VI no Recife ainda são escassos, podendo este trabalho servir como incentivo para contribuir com a melhoria na assistência farmacêutica do município.

\section{REFERÊNCIAS}

BRASIL. Portaria MS n. 1.271, de 6 de junho de 2014. Define a Lista Nacional de Notificação Compulsória de doenças, agravos e eventos de saúde pública nos serviços de saúde públicos e privados em todo o território nacional, nos termos do anexo, e dá outras providências. Diário Oficial da União, Brasília, DF, 6 de junho 2014. Disponível em: http://bvsms.saude.gov.br/bvs/saudelegis/gm/2014/prt1271_06_06_2014.html. Acesso em: 25 de novembro de 2016.

COÊLHO, Danieli Maria Matias et al. Perfil epidemiológico da tuberculose no Município de Teresina-PI, no período de 1999 a 2005 . Epidemiologia e Serviços de Saúde, v. 19, n. 1, p. 34-43, 2010.

SOGEBI, Olusola Ayodele et al. Limiares auditivos em pacientes com tuberculose resistente: configurações do audiograma basal e associações. Jornal Brasileiro de Pneumologia, v. 43, n. 3, p. 195-201, 2017. 